\title{
Effects of exercise on symptoms of anxiety, cognitive ability and sick leave in patients with anxiety disorders in primary care: study protocol for $\mathrm{PHYSBl}$, a randomized controlled trial
}

\author{
Jenny Nyberg ${ }^{1,2}$, Malin Henriksson $^{3}$, N. David Åberg ${ }^{4,5}$, Alexander Wall ${ }^{4,5}$, Robert Eggertsen ${ }^{3,6}$, Maria Westerlund ${ }^{6}$, \\ Louise Danielsson ${ }^{7,8}$, H. Georg Kuhn ${ }^{1,9}$, Margda Waern ${ }^{10,11}$ and Maria Åberg ${ }^{3,6^{*}}$ (D)
}

\begin{abstract}
Background: Anxiety disorders are common and associated with reduced quality of life, impaired physical and mental health and an increased economic burden for society. While evidence exists for the effectiveness of exercise treatment for depression, there is a need for high-quality randomized clinical trials (RCT) with a focus on anxiety disorders. Further research is also warranted regarding outcomes of cognitive function, other health-related variables, dose-response effects, work ability and potential mechanisms.

Method/design: Using a parallel, RCT design with three assessment points (baseline, post-intervention and oneyear follow-up), we aim to assess the effect of a 12-week exercise intervention in primary care patients with anxiety disorders $(n=180$ ), diagnosed using the Mini International Neuropsychiatric Interview (M.I.N.I; Swedish version 6.0.0d DSM-IV). Participants are randomly assigned to three physical exercise groups: one low-intensity training group, one moderate- to high intensity training group and one control non-exercise group. Assessments include measures of anxiety symptoms, cognitive function, physical health variables such as cardiovascular fitness, sick-leave and levels of hormones/cytokines in blood samples.
\end{abstract}

Discussion: Findings from this study will provide novel insights regarding the effects of exercise treatment on not only anxiety symptoms but also other outcomes including mental and physical health, cognitive function, doseresponse effects, work ability/sick leave and on biomarkers that may help explain underlying mechanisms.

Trial registration: The trial was registered at ClinicalTrial.gov NCT03247270 August 8, 2017.

Keywords: (n=3-10), Anxiety, Exercise, Cognitive function, Dose-response, Intervention

\section{Background}

Anxiety disorders are the most common mental disorders [1], with global prevalence figures ranging from 4 to $25 \%$ [2]. Data from the US indicate that one out of three will be affected by an anxiety disorder at some

\footnotetext{
* Correspondence: maria.aberg@gu.se

${ }^{3}$ Department of Public Health and Community Medicine/Primary Health Care, Institute of Medicine, Sahlgrenska Academy, University of Gothenburg, Box 454, SE-405 30 Gothenburg, Sweden

${ }^{6}$ Region Västra Götaland, Närhälsan, Gothenburg, Sweden

Full list of author information is available at the end of the article
}

point during lifetime [1]. In a national survey of the Swedish adult population, 36\% reported having anxiety issues [3] and lifetime prevalence for anxiety disorders in Sweden is estimated to be $25 \%$ [4]. In Sweden, it has been estimated that $70 \%$ of individuals who seek help for symptoms of anxiety and depression initially present in primary care [5] and costs for mental illness within primary care are on the rise [6].

Anxiety disorders substantially reduce quality of life and daily functioning [7] and are, among mental disorders, the second leading cause of years lived with

(c) The Author(s). 2019 Open Access This article is distributed under the terms of the Creative Commons Attribution 4.0 International License (http://creativecommons.org/licenses/by/4.0/), which permits unrestricted use, distribution, and 
disability [8]. They are also associated with elevated risks of cardiovascular disease [9] and premature mortality [10]. We have previously shown that anxiety disorders are associated with increased risk of future marginalization [11] as well as elevated mortality [12], emphasizing the importance of finding effective treatment strategies. Individuals with anxiety disorders frequently suffer from attentional problems, such as being easily distracted and unable to focus on ongoing tasks [13]. Moreover, impairments in cognitive function, including executive function and working memory, are often observed, which can be both a consequence and a symptom of anxiety [14-16]. Taken together, anxiety disorders place a considerable emotional, social, healthrelated and financial burden on both the individual and society.

The standard treatments for anxiety disorders include cognitive-behavioural therapy and pharmacological treatment [17]. Although these approaches often are effective in reducing anxiety symptoms, they may also be associated with treatment barriers and drawbacks. For example, nearly one third of patients do not respond to pharmacological treatment which may be associated with adverse side effects and noncompliance [17]. Patients may not wish to follow cognitive-behavioural therapy due to a reduced willingness to commit to treatment and stigmatization issues [18]. Collectively, these findings call for alternate complementary or stand-alone approaches to the treatment of anxiety disorders. Moreover, given the known association between anxiety disorders and cardiovascular disease, it is also essential to develop interventions that may target not only mental but also physical health problems in people with anxiety disorders. Exercise may represent an alternative, affordable and accessible treatment option for individuals with anxiety, both by itself and together with standard treatments. For clarification, exercise is a subset of physical activity that is planned, structured, repetitive and has a purpose of improving or maintaining physical fitness and physical activity is defined as any bodily movement produced by skeletal muscles that result in energy expenditure [19]. Physical fitness on the other hand is a set of attributes that are either health- or skill-related, including cardiovascular fitness and muscle strength.

Observational studies report an inverse association between physical activity and anxiety symptoms [20] and there is strong evidence for exercise in the treatment of depression [21]. Despite these findings, relatively little research has been focused on exercise in the treatment of anxiety. Nonetheless, meta-analyses of randomized controlled trials (RCTs) studying anxiolytic effects of exercise interventions in individuals with and without an anxiety diagnosis suggest that exercise is an effective treatment on its own, as well as combined with other therapies [22-26]. However, most of the interventions studies included in these meta-analyses are at high risk of bias. High-quality RCTs are needed to gain further understanding of the effects of exercise in treatment of anxiety [24] and to develop optimal exercise protocols.

Key gaps in the literature remain regarding the effects of exercise on other mental and physical health variables in patients with anxiety disorders including cognitive function, cardiovascular fitness, optimal exercise protocols, work ability/sick leave as well as the underlying mechanisms of the effect of exercise on anxiety symptoms. These are all issues that the current study aims to address. Given that cognitive function can be improved by exercise [27], the current study includes tests of executive function, creativity, response inhibition and cognitive flexibility. We will also evaluate the intervention effects on cardiovascular fitness, muscle strength, BMI and blood pressure. Further understanding of doseresponse relationships is important for establishing optimal protocols for exercise treatments in patients with anxiety, instead of only having to rely on general public health guidelines. Since dose-response effects of exercise have been minimally researched and studies of doseresponse are often inconclusive [22, 23], we include two intervention groups with separate intensity levels of exercise training. It is also highly relevant to evaluate the effects of exercise on work ability in patients with anxiety disorders, since reducing medical and sick-leave costs for anxiety disorders would liberate health care resources to be used elsewhere in an economically strained health care system. In order to gain further understanding regarding mechanistic aspects, treatment effect and prognosis, we will also investigate protein levels of trophic factors in blood samples, through which exercise may reduce anxiety. A broad spectrum of possible mechanisms exists, explaining the effect of exercise on anxiety including neurotransmitter, neuromodulator and psychological mechanisms [28], but the precise mechanism remains unclear.

We here propose an RCT study with varying intensity of exercise training in patients with anxiety disorders. To the best of our knowledge, this multifaceted study is the first study of its kind and it has the potential to provide novel and important contributions to existing scientific knowledge regarding how exercise may influence symptoms of anxiety, cognitive function, general health and work ability in patients with anxiety disorders.

\section{Research aims and hypothesis}

The primary aim is to investigate the effects of an exercise intervention on anxiety symptoms, cognitive function, physical health and quality of life and work ability in primary care patients who fulfil criteria for anxiety disorders. A secondary aim is to analyse any potential 
correlation of factors in blood samples (hormones/cytokines) with anxiety symptoms and function data and to study possible dose-response effects in patients assigned to exercise of different intensities. Another secondary aim is to study mediating or moderating effects of cardiovascular fitness or other comorbidities and to analyse possible differential effects in sub-groups of participants (different initial cardiovascular fitness levels, ages and gender). In addition, health-economic analyses will be performed concerning health-related quality of life effects associated with the intervention.

Our main hypothesis is that an exercise intervention will reduce symptoms of anxiety and the number of days on sick leave, improve cognitive function and physical health variables. An additional hypothesis is that the effect will be larger for high-intensity exercise than for low-intensity.

\section{Methods/design}

\section{Study design}

This is a randomized, parallel, controlled superiority clinical intervention study with three assessment points (baseline, post-intervention and one-year follow-up). The study is ongoing and was registered under ClinicalTrial.gov NCT03247270 on August 8, 2017 (https://clinicaltrials.gov/ct2/show/NCT03247270). The protocol was prepared in accordance with the SPIRIT 2013 statement. [29] Participants are randomly assigned to an exercise program of one low-intensity training group, one moderate- to high intensity training group and one control non-exercise group, with 1:1:1 allocation. Assessments and interventions are conducted at Primary Care Rehab (Närhälsan Primary Care) in Gothenburg, Sweden. Figure 1 shows the study design of the trial.

\section{Participants}

Individuals who seek help for anxiety issues at six primary care centres in southwest Sweden (Region Västra Götaland and Region Halland) are recruited. The patients are informed by their general practitioner (GP) or psychologist about the possibility of study participation and those expressing interest are provided with the study physician's phone number. Recruitment started in May 2017 and will continue until March 2020. Potential participants are then contacted by the study psychiatrist or nurse, who have no healthcare provider relationship to the patient.

Diagnoses and comorbidities are then determined by the study psychiatrist using the Mini International Neuropsychiatric Interview (M.I.N.I; Swedish version 6.0.0d DSM-IV), a short structured diagnostic interview with high reliability and validity [30]. Patients aged 18-65 diagnosed with anxiety disorders, including panic syndrome (F41.0), generalized anxiety (F41.1), mixed anxiety- and depression (F41.2 and F41.3), as well as anxiety NOS (F41.9) are included in the study.

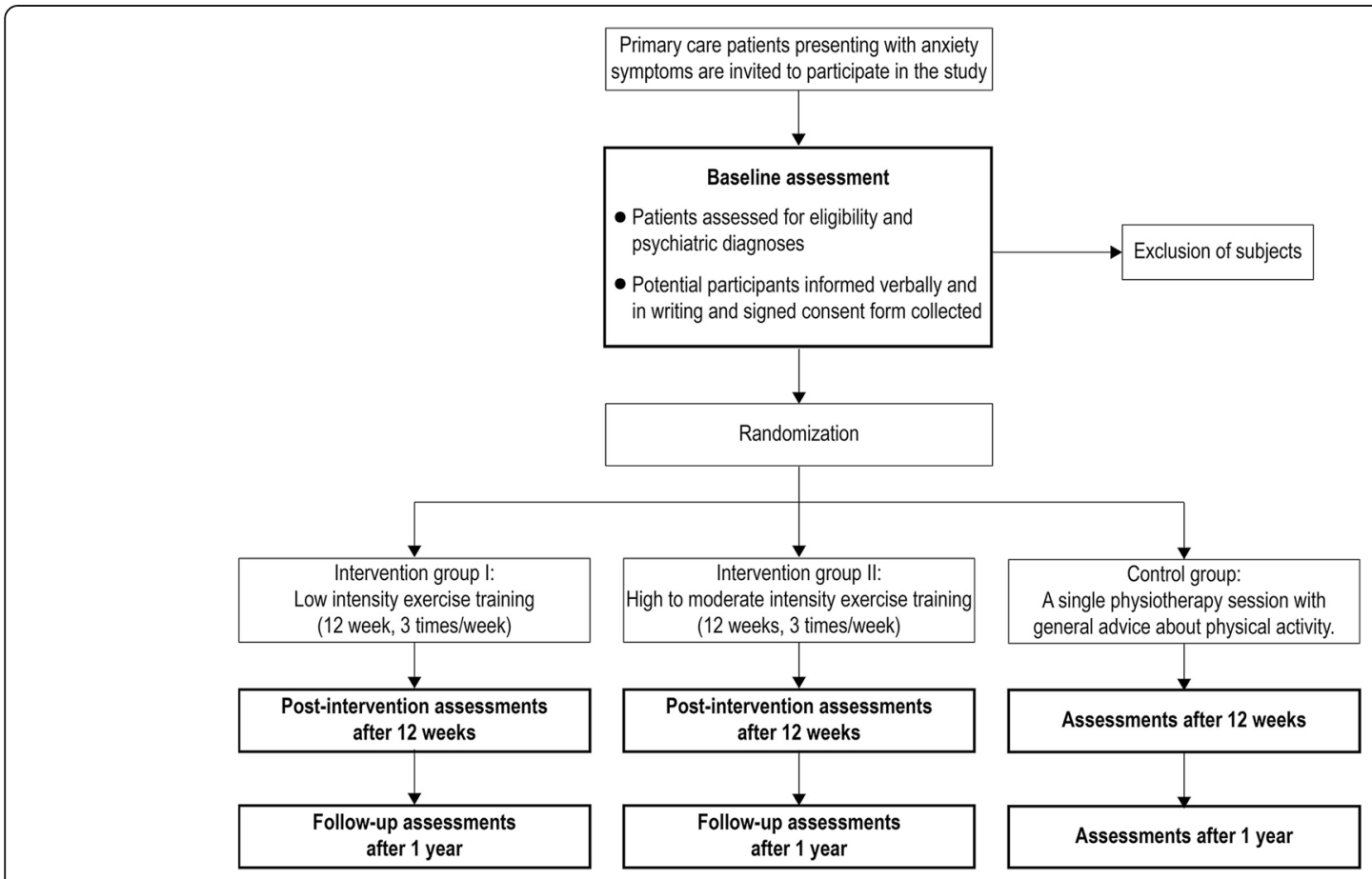

Fig. 1 Flow chart of study design 
Exclusion criteria include physical difficulties that preclude participation in the present exercise program, pathological electrocardiogram (ECG) including significant Q waves, ST-T changes, atrial fibrillation and left bundle-branch block, use of beta blockers, pregnancy, as well as previous psychiatric illness including bipolar disorder and psychotic disorders, ongoing substance abuse, ongoing burnout syndrome, and increased risk of suicide as assessed by the GP. An additional exclusion criterion is baseline physical activity level exceeding one exercise occasion per week. Individuals with and without ongoing treatment with psychoactive medication are included. Supportive contact, for example with a health center nurse, is not an exclusion criterion but individuals with ongoing psychotherapy are not included unless, after consultation with their GP, they can take a break in therapy for the duration of the 12-week intervention. Participant retention and adherence is promoted by exercise encouragement from the study physiotherapists, the possibility to receive personal results after study completion and reminders for follow-up assessment via letter, telephone and text message. The reasons for all eventual non-adherence and non-retentions will be documented.

\section{Intervention}

Using computer generated randomisation (http://www. randomizer.org), patients are randomized into three groups: 1) Intervention I: 12-week exercise program with low-intensity training 3 times per week for $45 \mathrm{~min}$; 2) Intervention II: 12-week exercise program with moderate to high-intensity training 3 times per week for 45 min; 3) Control group: a single physiotherapy session with general advice about physical activity. Participants are then informed by phone if they belong to an intervention group or the control group. Both intervention groups include cardiorespiratory and resistance training, and participants in group II are also encouraged to perform an additional running session per week. Participants in the control group are encouraged to start exercising after the study is completed and also receive a 3-months membership at a training facility, but are discouraged of actuating other exercise programs during the trial. All three groups self-report physical activity during the study. For participants in the intervention groups, the study physiotherapists designed individualized exercise programs during a one-to-one session with the patients on one occasion. The exercise program is designed as a circuit training with 12 stations, repeated twice ( $45 \mathrm{~s}$ workout, $15 \mathrm{~s}$ for transportation between stations and $1 \mathrm{~min}$ rest between the two laps). It also includes $10 \mathrm{~min}$ of warm-up exercises and 5-10 min of cool down and stretching exercises. Cardiorespiratory exercises include step-ups, lunges, jump rope, burpees, step touches side-to-side and step-touches on step board. Resistance training exercises include squats, abdominal plank position, hip lifts, crunches, row exercises and push-ups. Participants in intervention group I and II exercise separately in group sessions in the gym with the physiotherapist according to a predetermined schedule. A pulse-watch and the Borg RPE scale are used to monitor level of exertion to ensure appropriate exercise intensity. For intervention group I, the intensity level corresponds to 1.5-2.9 metabolic equivalents (METs), a Borg rated perceived exertion (RPE) of 10-14 and a maximal heart rate of $40-59 \%$. The corresponding figures for intervention group II are 3.0-8.9 METs, Borg RPE $12-17$ and $60-94 \%$ of maximal heart rate. Patients who are unable to participate in a session perform replacement exercises on their own.

\section{Blinding}

Researchers involved in data assessments (outcome assessors, study physicians, study psychologists, study psychiatrists, statistician and data collector) are blinded to the patients' intervention group. The only personnel aware of the treatment groups are the study physiotherapists who randomize the participants to the groups, code them, and also deliver the exercise interventions, but they are not involved in any data analyses. For half of the included patients, participation in low or high intensity training group was double-blind to both study researchers and patients: for the other half of the patients, training intensity group was only blind to the study researchers (the patients were aware of what treatment group they belonged to). Comparison of these two study halves will enable us to determine if blinding procedures reduce result bias. Final unblinding will occur after all assessments are performed and data collected at the 1year follow-up (June 2021).

\section{Assessments}

Assessments occur at baseline, post-intervention (after 12 weeks) and at one-year follow-up (Table 1). Participants will be assessed by the study physician, psychologist, psychiatrist and physiotherapist.

\section{Mental health variables}

Psychiatric diagnoses, determined by the study psychiatrist using M.I.N.I., are applied at initiation, at 12weeks post-intervention and 1-year follow-up. Severity of perceived symptoms of anxiety and depression are assessed using the established psychiatric selfassessment scales Beck Anxiety Inventory (BAI) [31] and Montgomery Åsberg Depression Rating Scale (MADRS) [32]. Patients report use of psychoactive prescription drugs, including dosage and duration at each assessment. 
Table 1 Measures, methods and assessment points used in the study

\begin{tabular}{|c|c|c|c|c|c|}
\hline Variable & Measure & Method & $\begin{array}{l}\text { T0 } \\
\text { Baseline }\end{array}$ & $\begin{array}{l}\mathrm{T} 1 \\
12 \text { weeks }\end{array}$ & $\begin{array}{l}\mathrm{T} 2 \\
1 \text { year }\end{array}$ \\
\hline Psychiatric diagnosis & M.I.N.I. & Interview & $x$ & $x$ & $x$ \\
\hline Anxiety symptoms & $\mathrm{BAl}$ & Self-report & $x$ & $x$ & $x$ \\
\hline Depression symptoms & MADRS & Self-report & $x$ & $x$ & $x$ \\
\hline Psychoactive drugs & Questionnaire & Self-report & $x$ & $x$ & $x$ \\
\hline Blood pressure & $\mathrm{MmHg}$ & Objective assessment & $x$ & $x$ & $x$ \\
\hline $\mathrm{BMI}$ & $\mathrm{Kg} / \mathrm{m}^{2}$ & Objective assessment & $x$ & $x$ & $x$ \\
\hline Alcohol use & AUDIT & Self-report & $x$ & $x$ & $x$ \\
\hline Quality of life & EQ-5D & Self-report & $x$ & $x$ & $x$ \\
\hline Cognitive function & WAIS-IV (d.s., b.d., m.r.) & Objective assessment & $x$ & $x$ & $x$ \\
\hline Cognitive function & D-KEFS (d.f.) & Objective assessment & $x$ & $x$ & $x$ \\
\hline Cardiovascular fitness & Åstrand's ergometer test & Objective assessment & $x$ & $x$ & $x$ \\
\hline Muscle strength & One-leg-rising-test & Objective assessment & $x$ & $x$ & $x$ \\
\hline Sick leave & Questionnaire & Self-report & $x$ & $x$ & $x$ \\
\hline Hormones/cytokines & S-IGF-1, S-BDNF, S-VEGF, CRP & Blood samples & $x$ & $x$ & $x$ \\
\hline
\end{tabular}

M.I.N.I. Mini International Neuropsychiatric Interview, BAI Beck Anxiety Inventory, MADRS Montgomery Åsberg Depression Rating Scale, BMI Body mass index, AUDIT Alcohol Use Disorders Identification Test, EQ-5D EuroQoL-5 Dimension Questionnaire, WAIS-IV Wechsler Adult Intelligence Scale, 4th edition, d.s. digit span, b.d. block design, m.r. matrix reasoning, D-KEFS Delis-Kaplan Executive Function System, d.f. design fluency, S-IGF-1 serum insulin-like growth factor 1, S-BDNF serum brain-derived neurotrophic factor, S-VEGF serum vascular endothelial growth factor, CRP high sensitivity C-reactive protein

\section{Cognitive function}

The Wechsler Adult Intelligence Scale, 4th edition (WAIS-IV) is used to measure executive functions and perceptual reasoning [33]. The digit span forward, backward and sequencing subtests were chosen to measure executive function, working memory, attention and mental manipulation. The block design and matrix reasoning subtests were chosen to measure visual-spatial processing and nonverbal problemsolving skills [33].

The Delis-Kaplan Executive Function System (DKEFS) is a standardized, non-verbal psychomotor test battery assessing executive functions in individuals between 8 and 89 years old [34]. The subtest Design fluency resembles the cognitive chain required in daily life to generate novel responses, while maintaining focus on a desired goal, and measures processes such as creativity, response inhibition, and cognitive flexibility [34, 35]. The Design fluency subtest consists of three conditions, all performed using a pen and paper where the participant has to bind together dots and make as many novel shapes possible during $60 \mathrm{~s}$. The participant needs to use working memory, inhibition and scanning skills to remember previously drawn shapes, inhibit the response to repeat shapes and scanning skills and creativity to find novel solutions to complete the assignment. The tasks appear in order of increasing difficulty, and the final trial includes a cognitive set-shifting component. D-KEFS has been used in both clinical and research settings showing good reliability and validity for measuring executive functions $[35,36]$.

\section{Physical health variables}

Blood pressure, weight and height are measured and body mass index (BMI) is calculated for each patient. Alcohol use is assessed using the Alcohol Use Disorders Identification Test (AUDIT) [37]. Patients self-report smoking, diet habits, concurrent illnesses, usage of prescribed drugs and physical activity in a questionnaire designed by the research team (Additional file 1). General health and perceived quality of life are measured using the EuroQoL-5 Dimension Questionnaire (EQ-5D), a standardized instrument measuring health related to five dimensions: mobility, self-care, usual activities, pain/discomfort and anxiety/depression [38].

\section{Cardiovascular fitness}

Maximal oxygen uptake capacity (VO2max) is estimated using Åstrand's submaximal ergometer test and serves as a measure of cardiovascular fitness [39]. The test is performed by the physiotherapists at Primary Care Rehab. Participants are told to refrain from vigorous activity the day before the test, to avoid a heavy meal within three hours and smoking/snuff use one hour prior to the test and also to avoid stress during the test day. The ergometer is calibrated for each participant and heart rate is corrected for sex and age. Predicted VO2max is calculated in accordance with the nomogram described by Åstrand and Ryhming [39].

\section{Muscle strength}

Muscle strength is measured using a standardized test of maximum number of one-leg rises from sitting on a 
chair (45 cm high) during $1 \mathrm{~min}$ [40]. The patient is told to keep the arms crossed and to hold the other leg extended without touching the floor. The test is performed with full muscle control and at constant speed without adding arm or trunk movements.

\section{Work ability}

Patients self-report work ability, weekly number of work hours and if they are on sick-leave in a questionnaire (Additional file 2).

\section{Blood samples}

Blood samples are taken by a biomedical analyst to investigate any potential correlation of the following hormones/cytokines with anxiety symptoms and function data: S-insulin-like growth factor 1 (IGF-1), S-brainderived neurotrophic factor (BDNF), S-vascular endothelial growth factor (VEGF) and high sensitivity Creactive protein (CRP). Total accumulated blood volume is $15 \mathrm{ml}$ per individual. The blood samples are coded and stored in a separate, locked freezer ( -70 degrees) at Sahlgrenska University Hospital, Gothenburg. All analyses will be performed in collaboration with the central laboratory at Sahlgrenska University Hospital, Gothenburg. After study completion, the samples will be destroyed. The Swedish Health and Social Care Inspectorate (IVO), a government agency under the Ministry of Health and Social Affairs, has been notified of the establishment of a biobank (No 946).

\section{Sample size calculation/power calculation}

A power analysis was performed by a statistician prior to study initiation. We have calculated, based on other studies [41, 42], that an effect size of 0.5 difference between the groups is clinically relevant, which equates to a 5-point difference between the groups on the BAI scale. Standard deviations from a previous study using BAI as the outcome measurement were in the interval of 5-10 [43]. We use SD 9 for our calculations. Based on these assumptions, we should include 60 patients in each of the treatment arms to reach $80 \%$ power at the $5 \%$ significance level. In order to achieve an adequate number of study participants, we will initially recruit $25 \%$ more subjects than required.

\section{Statistical analysis}

Data will be presented using descriptive statistics, including the number of observations, means and standard deviations for continuous variables. For categorical variables, frequencies and percentages will be presented and data analysed using Chi-squared tests. Based on plotted histograms (confirming that the data may be considered as approximately normally distributed), continuous data will be analysed using a univariate general linear model
(ANCOVA). In this model, treatment will be used as a categorical independent variable and baseline levels as covariates. Data will be presented as changes of means with corresponding 95\% confidence intervals, standard deviations and $p$-values from group comparisons. The categorical outcome "treatment responsive" will be analysed using Chi-squared tests. A p-value below 0.05 will be considered statistically significant for all analyses. All statistical analyses will be performed by a statistician using SAS version 9.4 (SAS Institute, Cary, NC).

\section{Drop-out analyses}

Drop-out analysis is a type of sensitivity analysis where we aim to examine differences between drop-outs and participants of all study groups concerning several descriptive variables (age, gender, BMI, cardiovascular fitness, perceived anxiety level, cognitive function, comorbidity) in order to investigate if the incidence of drop-outs is selective or random.

Outcome data collected prior to an eventual later study discontinuation will be included in the analyses i.e. a participant lacking data from the 1-year follow-up will still have data from the 12-week post-intervention.

\section{Health-economic analyses}

Health-economic analyses will be performed using primary data generated within the project concerning health-related quality of life effects associated with the intervention and information about healthcare resources utilized. A health-economic model that depicts the clinical trial will be constructed and used for computing cost-utility measures. Moreover, the model will be constructed for performing extensive probabilistic sensitivity analyses $[44,45]$.

\section{Project timeline}

Recruitment started in May 2017 and will continue until March 2020. The trial is divided into one pilot set and five trial sets and the estimated primary completion date is June, 2021 (Fig. 2).

\section{Ethical considerations}

The study is approved by the regional Ethics Committee in the Gothenburg, Sweden, National Board of Health prior to start (300-16) prior to start.

Interested patients are informed verbally and in writing about the aims and the study design including randomization, the importance of active participation for reliable data but also about the possibility to withdraw from the study at any time without disadvantages. Each participant has to sign a statement of informed consent, delivered by the study physician, before study inclusion.

All data collected will be coded and anonymized. A key connecting serial numbers with personal 


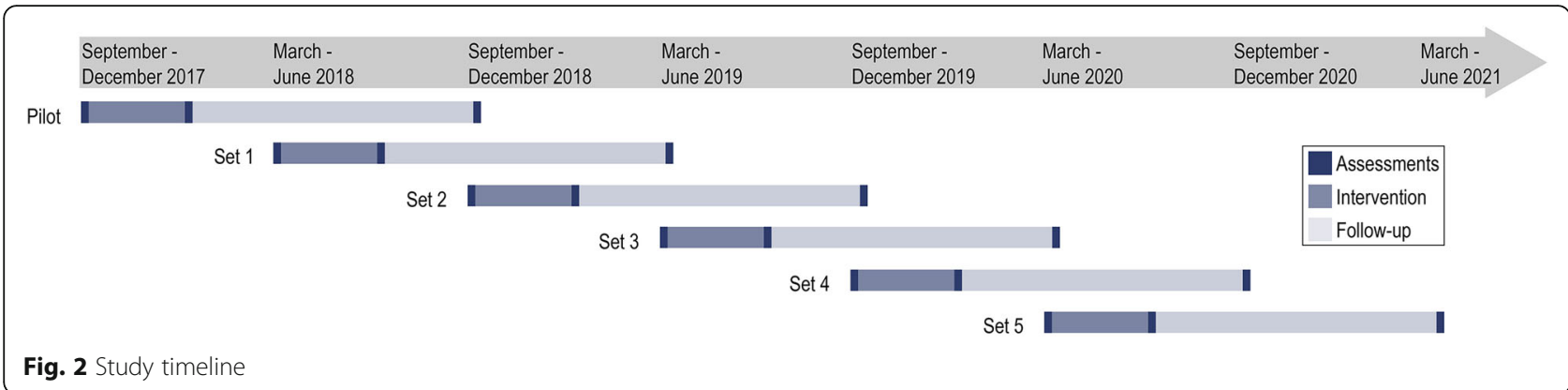

identification numbers will be stored and locked away. All analyses will be performed on coded data presented on group levels. The database will be passwordprotected, encrypted and stored for 10 years in a locked, fireproof archive at Gothenburg University, designed for such purposes. All participating researchers will have access to the database. Collected blood samples will be destroyed after the completion of the study. A Data Protection Officer (DPO) has been assigned and reported to the Swedish Data Protection Authority.

The current research team has previously performed an intervention study on the effects of exercise on depressed patients [46] and hence anticipates low risk of complications. A small risk for injury while exercising exists, such as a sprained ligament or a strained muscle. In order to minimize the risk for injuries, exercise programs are developed and modified according to the ability of each patient by experienced primary care physiotherapists. Moreover, ECG is monitored before the intervention and individuals with a pathological ECG will be excluded, which will minimize the risk for cardiac side-effects during exercise.

In order to reduce the risk for worsening mental health or symptoms during the study we have a) assessed eligibility for study participation by a physician; b) excluded patients with heightened suicide risk; c) requested participants to contact research personnel if they feel worse; d) as intervention personnel, only included persons with knowledge regarding symptoms of worsening of anxiety and/or depression. If a patient experience worsening of symptoms, the subject will be excluded from the study and put in contact with the treating physician or psychologist. The study physician and physiotherapists will continuously inquire about and record any signs of worsening of mental health symptoms or side-effects.

Another experience from our previous intervention study on depression was the disappointment of being randomized to the control group. Therefore, the patients in the control group will have a single session with a physiotherapist and general advice about physical activity according to public health recommendations as well as a 3-month membership at a training facility for after study completion. Such a control condition, was chosen to potentially feel meaningful and inspiring to the participants. [47] A passive control group such as a wait-list design or treatment as usual are associated with negative influences on study results for nonpharmacological treatments, including a possible overestimation of the intervention effects and limited blinding possibilities. [48] Instead of an active control with an alternative behaviour, the current study has two separate interventions groups with different exercise intensities, a design that allows for comparison effectiveness depending on exercise dose as well as permits blinding for participants. All study participants will be informed about the results from the study, as will all participating professionals and the general medical community. Results will be published in peer-reviewed medical journals and results will be disseminated regardless of the magnitude or direction of effect. When writing manuscripts, ICMJE (International Committee of Medical Journal Editors) guidelines regarding authorship will be followed.

\section{Discussion}

This is an extensive intervention study (RCT) exploring the effects of exercise training in primary care patients with anxiety. The unique study design with several strengths will provide novel insights and include outcome measures not only for anxiety symptoms, but also for a broad range of areas including mental and physical health, cognitive function, dose-response effects, work ability/sick leave and underlying mechanisms. If this exercise trial proves beneficial, this study will provide new directions for primary care service regarding prevention and treatment for persons with anxiety disorders.

\section{Additional files}

Additional file 1: Questionnaire PHYSBI. (PDF $111 \mathrm{~kb}$ )

Additional file 2: Work ability PHYSBI. (PDF $81 \mathrm{~kb}$ )

\section{Abbreviations}

AUDIT: Alcohol Use Disorders Identification Test; BAl: Beck Anxiety Inventory; BDNF: Brain-derived neurotrophic factor; BMI: Body mass index; CRP: Creactive protein; D-KEFS: The Delis-Kaplan Executive Function System; ECG: Electrocardiogram; EQ-5D: The EuroQoL-5 Dimension Questionnaire; 
GP: General practitioner; IGF-1: Insulin-like growth factor 1; M.I.N.I.: Mini International Neuropsychiatric Interview; MADRS: Montgomery Åsberg Depression Rating Scale; MTE: Metabolic equivalents; RCT: randomized controlled trial; RPE: Borg rated perceived exertion; VEGF: Vascular endothelial growth factor; VO2max: Maximal oxygen uptake capacity; WAIS-IV: The Wechsler Adult Intelligence Scale, 4th edition

\section{Acknowledgements}

The authors would like to thank Annika Appelgren at Närhälsan Askim, Region Västra Götaland for support and encouragement. We also thank Helena Cato and all involved physiotherapists and Actimera Health Club, Kungsbacka. Special thanks are given to Marion Walser at the Institute of Medicine, Sahlgrenska Academy, University of Gothenburg, Birgitta Johansson at the Institute of Neuroscience and Physiology, Sahlgrenska Academy, University of Gothenburg, Ulla Karilampi, Emelie Delphin and Stefan Wiktorsson at the Department of Psychiatry and Neurochemistry, Institute of Neuroscience and Physiology, Sahlgrenska Academy, University of Gothenburg, Sweden and Linus Schiöler at the Department of Public Health and Community Medicine, Institute of Medicine, Sahlgrenska Academy, University of Gothenburg.

\section{Authors' contributions}

$\mathrm{MH}$ and $\mathrm{M} \AA$ conceptualized and $\mathrm{MH}, \mathrm{M} \AA, \mathrm{RE}, \mathrm{HGK}$ and $\mathrm{MW} 2$ designed the study. MW1, DÅ, AW and LD contributed to data acquisition. MÅ, DÅ, AW and HGK planned the statistical analyses. JN drafted the work and all coauthors contributed to interpretation and critically revising the manuscript for important intellectual content. All authors have read and approved this final version and are guarantors.

\section{Funding}

Funding sources include grants from the Swedish state under the agreement between the Swedish Government and the county councils, the ALF-agreement [ALFGBG-813511, ALFGBG-715841, ALFGBG-726541] and the Region of Västra Götaland, Gothenburg, Sweden, Grant no. VGFOUREG$645151 ; 734131 ; 841361$. This study protocol has undergone peer-review by the Swedish governmental funding body. Funding is only financial and independent of study design, collection, analysis and interpretation of data, writing the study protocol or decision to submit.

\section{Availability of data and materials}

The database generated for the current study is not publicly available for ethical reasons but data on group level are available from the corresponding author on reasonable request.

\section{Ethics approval and consent to participate}

The study is approved by the regional Ethics Committee in the Gothenburg, Sweden, National Board of Health prior to start (300-16). Participants are informed about the aims and the study design including randomization and about the possibility of ending their participation at any time without disadvantages. Each participant has signed a statement of informed consent before study inclusion.

\section{Consent for publication}

Not applicable as we do not provide personal information for publication.

\section{Competing interests}

The authors declare that they have no competing interests.

\footnotetext{
Author details

${ }^{1}$ Center for Brain Repair and Rehabilitation, Institute of Neuroscience and Physiology, Sahlgrenska Academy, University of Gothenburg, Gothenburg, Sweden. ${ }^{2}$ Neurology Clinic, Sahlgrenska University Hospital, Region Västra Götaland, Gothenburg, Sweden. ${ }^{3}$ Department of Public Health and Community Medicine/Primary Health Care, Institute of Medicine, Sahlgrenska Academy, University of Gothenburg, Box 454, SE-405 30 Gothenburg, Sweden. ${ }^{4}$ Department of Internal Medicine, Institute of Medicine, Sahlgrenska Academy, University of Gothenburg, Gothenburg, Sweden. ${ }^{5}$ Department of Internal Medicine, Sahlgrenska University Hospital, Region Västra Götaland, Gothenburg, Sweden. ${ }^{6}$ Region Västra Götaland, Närhälsan, Gothenburg, Sweden. ${ }^{7}$ Department of Health and Rehabilitation, Institute of Neuroscience and Physiology, Sahlgrenska Academy, University of Gothenburg,
}

Gothenburg, Sweden. ${ }^{8}$ Angered Hospital, Region Västra Götaland, Gothenburg, Sweden. ${ }^{9}$ Center for Stroke Research and Neurocure Cluster of Excellence, Charité - Universitätsmedizin Berlin, Berlin, Germany. ${ }^{10}$ Department of Psychiatry and Neurochemistry, Institute of Neuroscience and Physiology, Sahlgrenska Academy, University of Gothenburg, Gothenburg, Sweden. "1"Psychosis Clinic, Sahlgrenska University Hospital, Region Västra Götaland, Gothenburg, Sweden.

Received: 15 May 2019 Accepted: 31 May 2019

Published online: 10 June 2019

\section{References}

1. Kessler RC, Petukhova M, Sampson NA, Zaslavsky AM, Wittchen HU. Twelvemonth and lifetime prevalence and lifetime morbid risk of anxiety and mood disorders in the United States. Int J Methods Psychiatr Res. 2012; 21(3):169-84.

2. Remes $\mathrm{O}$, Brayne $\mathrm{C}$, van der Linde $\mathrm{R}$, Lafortune $\mathrm{L}$. A systematic review of reviews on the prevalence of anxiety disorders in adult populations. Brain Behav. 2016;6(7):e00497.

3. Socialstyrelsen. Tillståndet och utvecklingen inom hälso- och sjukvård samt tandvård. Lägesrapport Rapport Stockholm. 2016:2016-3-16.

4. Socialstyrelsen. Öppna jämförelser 2015: hälso- och sjukvård vid kroniska sjukdomar. In: Stockholm; 2015

5. Socialstyrelsen. Hälso- och sjukvård - primärvård. lägesrapport Stockholm. 2007:2007-131-13

6. Socialstyrelsen. Nationell utvärdering 2013 - vård och insatser vid depression, ångest och schizofreni. Rekommendationer, bedömningar och sammanfattning. . Stockholm. 2013;2013-6-27.

7. Buist-Bouwman MA, De Graaf R, Vollebergh WA, Alonso J, Bruffaerts R, Ormel J, et al. Functional disability of mental disorders and comparison with physical disorders: a study among the general population of six European countries. Acta Psychiatr Scand. 2006;113(6):492-500

8. Whiteford HA, Degenhardt L, Rehm J, Baxter AJ, Ferrari AJ, Erskine HE, et al. Global burden of disease attributable to mental and substance use disorders: findings from the global burden of disease study 2010. Lancet. 2013

9. Batelaan NM, Seldenrijk A, Bot M, van Balkom AJ, Penninx BW. Anxiety and new onset of cardiovascular disease: critical review and meta-analysis. $\mathrm{Br} J$ Psychiatry. 2016;208(3):223-31.

10. Tolmunen T, Lehto SM, Julkunen J, Hintikka J, Kauhanen J. Trait anxiety and somatic concerns associate with increased mortality risk: a 23-year follow-up in aging men. Ann Epidemiol. 2014;24(6):463-8.

11. Love J, Hensing G, Soderberg M, Toren K, Waern M, Aberg M. Future marginalisation and mortality in young Swedish men with non-psychotic psychiatric disorders and the resilience effect of cognitive ability: a prospective, population-based study. BMJ Open. 2016:6(8):e010769.

12. Henriksson M, Nyberg J, Schioler L, Hensing G, Kuhn GH, Soderberg M, et al. Cause-specific mortality in Swedish males diagnosed with non-psychotic mental disorders in late adolescence: a prospective population-based study. J Epidemiol Community Health. 2018;72(7):582-8.

13. Vytal KE, Cornwell BR, Letkiewicz AM, Arkin NE, Grillon C. The complex interaction between anxiety and cognition: insight from spatial and verbal working memory. Front Hum Neurosci. 2013;7:93.

14. Ferreri F, Lapp LK, Peretti CS. Current research on cognitive aspects of anxiety disorders. Curr Opin Psychiatry. 2011;24(1):49-54.

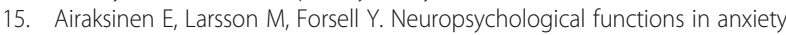
disorders in population-based samples: evidence of episodic memory dysfunction. J Psychiatr Res. 2005;39(2):207-14.

16. Balderston NL, Vytal KE, O'Connell K, Torrisi S, Letkiewicz A, Ernst M, et al. Anxiety patients show reduced working memory related dIPFC activation during safety and threat. Depress Anxiety. 2017;34(1):25-36.

17. Baldwin DS, Anderson IM, Nutt DJ, Allgulander C, Bandelow B, den Boer JA, et al. Evidence-based pharmacological treatment of anxiety disorders, posttraumatic stress disorder and obsessive-compulsive disorder: a revision of the 2005 guidelines from the British Association for Psychopharmacology. J Psychopharmacol. 2014;28(5):403-39

18. Overton SLMSL. The stigma of mental illness. Journal of Counseling \& Development. 2008:86:143-56.

19. Caspersen CJ, Powell KE, Christenson GM. Physical activity, exercise, and physical fitness: definitions and distinctions for health-related research. Public Health Rep. 1985;100(2):126-31. 
20. Strickland JC, Smith MA. The anxiolytic effects of resistance exercise. Front Psychol. 2014:5:753.

21. Cooney GM, Dwan K, Greig CA, Lawlor DA, Rimer J, Waugh FR, et al. Exercise for depression. Cochrane Database Syst Rev. (2013, 9):CD004366.

22. Wipfli BM, Rethorst CD, Landers DM. The anxiolytic effects of exercise: a meta-analysis of randomized trials and dose-response analysis. J Sport Exerc Psychol. 2008;30(4):392-410.

23. Jayakody K, Gunadasa S, Hosker C. Exercise for anxiety disorders: systematic review. Br J Sports Med. 2014;48(3):187-96.

24. Stubbs B, Vancampfort D, Rosenbaum S, Firth J, Cosco T, Veronese N, et al. An examination of the anxiolytic effects of exercise for people with anxiety and stress-related disorders: a meta-analysis. Psychiatry Res. 2017;249:102-8.

25. Aylett $E$, Small N, Bower P. Exercise in the treatment of clinical anxiety in general practice - a systematic review and meta-analysis. BMC Health Serv Res. 2018;18(1):559.

26. Stonerock GL, Hoffman BM, Smith PJ, Blumenthal JA. Exercise as treatment for anxiety: systematic review and analysis. Ann Behav Med. 2015;49(4):542-56.

27. Colcombe $S$, Kramer AF. Fitness effects on the cognitive function of older adults: a meta-analytic study. Psychol Sci. 2003;14(2):125-30.

28. DeBoer LB, Powers MB, Utschig AC, Otto MW, Smits JA. Exploring exercise as an avenue for the treatment of anxiety disorders. Expert Rev Neurother 2012;12(8):1011-22.

29. Chan AW, Tetzlaff JM, Gotzsche PC, Altman DG, Mann H, Berlin JA, et al. SPIRIT 2013 explanation and elaboration: guidance for protocols of clinical trials. BMJ. 2013;346:e7586.

30. Sheehan DV, Lecrubier $Y$, Sheehan KH, Amorim P, Janavs J, Weiller E, et al. The Mini-International Neuropsychiatric Interview (M.I.N.I.): the development and validation of a structured diagnostic psychiatric interview for DSM-IV and ICD-10. J Clin Psychiatry. 1998;59 Suppl 20:22-33;quiz 4-57.

31. Beck AT, Epstein N, Brown G, Steer RA. An inventory for measuring clinical anxiety: psychometric properties. J Consult Clin Psychol. 1988;56(6):893-7.

32. Montgomery SA, Asberg M. A new depression scale designed to be sensitive to change. Br J Psychiatry. 1979;134:382-9.

33. Wechsler D. Wechsler Adult Intelligence Scale-Fourth Edition. San Antonio: TX: Psychological Corporation; 2008

34. Delis DC, Kaplan E, Kramer JH. Delis-Kaplan executive function system (DKEFS) examiner's manual. In: The psychological corporation. San Antonio, Texas, USA; 2001.

35. Homack S, Lee D, Riccio CA. Test review: Delis-Kaplan executive function system. J Clin Exp Neuropsychol. 2005;27(5):599-609.

36. Karr JE, Hofer SM, Iverson GL, Garcia-Barrera MA. Examining the latent structure of the Delis-Kaplan executive function system. Arch Clin Neuropsychol. 2018.

37. Saunders JB, Aasland OG, Babor TF, de la Fuente JR, Grant M. Development of the alcohol use disorders identification test (AUDIT): WHO collaborative project on early detection of persons with harmful alcohol consumption--II. Addiction. 1993;88(6):791-804.

38. EuroQol G. EuroQol--a new facility for the measurement of health-related quality of life. Health Policy. 1990;16(3):199-208.

39. Åstrand PO, Rodahl K, Dahl HA, Stromme SB. Textbook of work physiology: physiological bases of exercise. 4th ed: Champaign (III.) : human kinetics, 2003.; 2003.

40. Larsson AC, Petersson I, Ekdahl C. Functional capacity and early radiographic osteoarthritis in middle-aged people with chronic knee pain. Physiother Res Int. 1998;3(3):153-63.

41. Shiranibidabadi S, Mehryar A. Music therapy as an adjunct to standard treatment for obsessive compulsive disorder and co-morbid anxiety and depression: a randomized clinical trial. J Affect Disord. 2015;184:13-7.

42. Usmani ZA, Carson KV, Heslop K, Esterman AJ, De Soyza A, Smith BJ. Psychological therapies for the treatment of anxiety disorders in chronic obstructive pulmonary disease. Cochrane Database Syst Rev. 2017;(3): CD010673.

43. Cully JA, Stanley MA, Petersen NJ, Hundt NE, Kauth MR, Naik AD, et al. Delivery of brief cognitive behavioral therapy for medically ill patients in primary care: a pragmatic randomized clinical trial. J Gen Intern Med. 2017; 32(9):1014-24.

44. Drummond MF, Sculpher MJ, Claxton K, Stoddart GL, Torrance GW. Methods for the economic evaluation of health care Programmes, 4th edition. Publications OM: Oxford University Press; 2015.

45. Gray AM. Applied methods of cost-effectiveness analysis in healthcare (handbooks in health economic evaluation book 3) 1st edition. 1st ed. USA: Oxford University Press; 2010.
46. Danielsson L, Papoulias I, Petersson EL, Carlsson J, Waern M. Exercise or basic body awareness therapy as add-on treatment for major depression: a controlled study. J Affect Disord. 2014;168:98-106.

47. Aberg MA, Nyberg J, Toren K, Sorberg A, Kuhn HG, Waern M. Cardiovascular fitness in early adulthood and future suicidal behaviour in men followed for up to 42 years. Psychol Med. 2013:1-10.

48. Kinser PA, Robins JL. Control group design: enhancing rigor in research of mind-body therapies for depression. Evid Based Complement Alternat Med. 2013:2013:140467.

\section{Publisher's Note}

Springer Nature remains neutral with regard to jurisdictional claims in published maps and institutional affiliations.
Ready to submit your research? Choose BMC and benefit from:

- fast, convenient online submission

- thorough peer review by experienced researchers in your field

- rapid publication on acceptance

- support for research data, including large and complex data types

- gold Open Access which fosters wider collaboration and increased citations

- maximum visibility for your research: over $100 \mathrm{M}$ website views per year

At $\mathrm{BMC}$, research is always in progress.

Learn more biomedcentral.com/submissions 